\title{
Una mirada desde la ingeniería industrial al uso de herramientas tecnológicas en la Administración Pública
}

A look from the industrial engineering to the use of technological tools in the Public Administration

\section{ANA MERCEDES FRAILE-BENÍTEZ}

Ingeniera Industrial

Mg. MBA Dirección y Administración de Empresas

Grupo de Investigación LOGyCA

Universidad de Boyacá, Colombia

anafraile@uniboyaca.edu.co 


\title{
RESUMEN
}

El presente artículo de reflexión, tiene como objetivo principal mostrar cómo las herramientas tecnológicas diseñadas para un uso específico pueden ser utilizadas con recursividad para la gestión tecnológica. De esta manera se argumenta la importancia de usar herramientas tecnológicas en la solución de problemas en la administración pública, donde generalmente los recursos son escasos, las necesidades en relación al manejo de información abundan y las restricciones para adquisición de tecnología aumentan; se requiere por parte de los profesionales habilidades para administrar bajo principios de eficacia, eficiencia y transparencia. Se describe el uso dado a las herramientas tecnológicas en dos organizaciones públicas y se muestra cómo en los dos casos el uso de portales y diversos recursos tecnológicos permiten a la administración realizar procesos de planeación y mantenimiento del sistema de mejora continua. Se muestra inicialmente el caso del Municipio de Paipa en lo relativo al proceso de planeación articulado a los Objetivos de Desarrollo del Milenio y el Plan de Desarrollo; posteriormente se presenta el uso dado por el Centro de Formación de Talento Humano en Salud (CFTHS) del Servicio Nacional de Aprendizaje (SENA) a las herramientas tecnológicas en su sistema de mejora continua. Finalmente se plantea el punto de vista que permita al lector identificar el actuar del Ingeniero Industrial.

Palabras clave: Gestión Pública, Ingeniería Industrial, Herramientas Tecnológicas.

\begin{abstract}
This reflection article is aimed to show how the technological tools designed for a specific application can be used with recursion for the technology management. Argues the importance of using technological tools in the solution of problems in the public administration, where the resources are generally scarce, needs in relation to the handling of information abound and restrictions for acquisition of technology increase; skills to manage under principles of effectiveness, efficiency and transparency are required by the professionals. It describes the use given to the technological tools in two public organizations and shows how in both cases the use of portals and various technological resources allow the management of the company to make planning and maintenance processes of the system of
\end{abstract}


continuous improvement. The case of the municipality of Paipa is initially displayed in relation to the planning process articulated to the Millennium Development Goals and the Municipal Plan of Development; subsequently taught the use of technological tools in its system of continuous improvement, given by the Training Centre of Human Resource in Health (CFTHS) of the National Learning Service (SENA). Finally, it presents a point of view that allows the reader to identify acting of the Industrial Engineer.

Keywords: Public Management, Industrial Engineering, Quality Management. 


\section{INTRODUCCIÓN}

El concepto de Gestión Pública dada por el Departamento Nacional de Planeación DNP (2014) se establece como el proceso dinámico, integral, sistemático y participativo, que articula la planificación, ejecución seguimiento, evaluación, control y rendición de cuentas de las estrategias de desarrollo económico, social, cultural, tecnológico, ambiental, político e institucional de una administración, sobre la base de metas asociadas a la democracia.

También es necesario considerar que la Gestión Pública, según lo establecido por la Escuela Superior de Administración Pública - ESAP (2014), implica gerenciar políticas públicas, programas de gobierno e instituciones públicas, que respondan a las necesidades políticas, económicas y sociales, liderar procesos de cambio institucional que permitan la modernización del Estado y la Gestión Pública, acordes con el cambio social, conocer las áreas precisas de acción que el Estado debe adelantar a través de sus agencias.

De otra parte el Departamento Administrativo de la Función Pública, indica que la gestión pública es el conjunto de actividades desarrolladas por los organismos del Estado para planear, ejecutar y evaluar la atención de necesidades y la solución a problemas de las comunidades, mediante la generación de bienes y prestación de servicios sobre la base de metas que deben ser acordadas democráticamente. Concretamente indica que la Gestión se refiere a las actividades y lo público se refiere a lo que es común, a que es de todos y para todos.

Asimismo, tal como lo expone Alzate, J. (2009) en el libro "Capital social, descentralización y modernización del estado", existe la gestión pública que puede estar basada en resultados, en el ciclo del proyecto, orientada al desempeño o a la gestión documental, todas estas como actividades coordinadas para planificar, controlar, asegurar y mejorar una entidad.

En este orden de ideas y especialmente en lo que tiene que ver con gestión documental, la entrega constante y permanente de información pública se convierte en una tarea que congestiona las actividades de los funcionarios públicos.

El uso de herramientas tecnológicas aplicadas a procesos de mejoramiento continuo se enmarca en los proyectos gerenciales más importantes para la alta dirección; por lo anterior se presenta un contexto teórico relacionado con la tecnología, para entender la situación problema que se orienta a generar un desarrollo reflexivo sobre actividades de mejora en el sector público colombiano, mostrando desde una perspectiva interpretativa importantes acciones de quienes lideraron los procesos y entidades del estado. 


\section{LA TECNOLOGÍA Y LO PÚBLICO.}

\section{Las herramientas tecnológicas}

Inicialmente es necesario considerar lo relacionado a Gestión Tecnológica y para ello se presenta lo expuesto por Núñez (2011), quien muestra cómo incide la tecnología en la gestión, mencionando entre otros estudios, los realizados por el Grupo de Gestión de la Tecnología GETEC (2008) de la Universidad Politécnica de Madrid, argumentando que la tecnología es una poderosa herramienta, la cual debe estar enmarcada en los procesos generales de innovación a los que están sometidas las organizaciones; adicionalmente, referencia lo expuesto por Escorsa y Valls (2005), quienes indican que la gestión tecnológica juega un papel fundamental en la coordinación, e integración de las diversas funciones directivas, gestión de la investigación y desarrollo, concluyendo que es un elemento fundamental para la gestión organizacional. Desde otro ángulo y en lo relacionado con la adecuación al uso, Sequera (1998) plantea que las nuevas formas de tener acceso a la información han creado oportunidades para repensar, revisar y practicar el aprendizaje en modos en que hasta ahora eran logística y económicamente imposibles.

Con respecto a la tecnología y según lo plasmado por Jaramillo (1999), se identifica que la tecnología puede ser vista como un conjunto de conocimientos utilizados para la producción y comercialización de bienes y servicios; asimismo considera a las herramientas tecnológicas como aquellas que facilitan la realización de procesos y permiten el intercambio de información y conocimiento dentro y fuera de las organizaciones.

Se contempla entonces a las herramientas tecnológicas como fundamentales para aumentar la eficacia y eficiencia en los procesos de comunicación y manejo de información de las diferentes organizaciones, razón por la cual han cobrado mayor sentido y son utilizadas en procesos de mejoramiento continuo ya que agregan valor y puntualizan lo relacionado con la información en el planear - hacer - verificar, tres de los componentes fundamentales en el ciclo de mejora continua expuesto por Deming (1982), para posteriormente soportar ejercicios de revisión y análisis, garantizando la toma de decisiones en lo referente al último componente del ciclo, correspondiente al "actuar".

\section{Uso de herramientas tecnológicas en dos entidades del sector público colombiano}

La norma técnica de gestión pública NTC GP 1000:2009 articula el Sistema de Control Interno, el Sistema de Desarrollo Administrativo y el Sistema de Gestión de la Calidad, establece las generalidades y los requisitos mínimos para definir, documentar, implementar y mantener un Sistema de Gestión de la Calidad en los organismos, entidades y agentes obligados conforme al artículo $2^{\circ}$ de la Ley 872 de 2003. Los responsables de implementar los sistemas de gestión presentan las dificultades propias 
del manejo de información, especialmente si ésta se documenta de manera tradicional; igualmente el presupuesto público no siempre es suficiente y por lo tanto no se asigna rubro para la actualización tecnológica, porque se priorizan otros sectores de inversión, razón por la cual, quienes tienen el reto de mostrar resultados de gestión en un periodo de cuatro años o menos, buscan alternativas para agregar valor a sus procesos tomando decisiones para presentar resultados en términos de eficacia, eficiencia y efectividad, sin mayor impacto, ya que llevan una década buscando estrategias que les permitan alcanzar la implementación de sus sistemas de gestión con dificultades en los procesos de medición, análisis y mejora.

A continuación se presenta la experiencia relacionada con el manejo de información en procesos de planeación y mejora continua en dos entidades públicas, en donde se dio uso a las herramientas tecnológicas asegurando la oportunidad, la comunicación, la disponibilidad y repetibilidad de información.

La primera de ellas se desarrolla en la Administración Municipal de Paipa, municipio del departamento de Boyacá, Colombia, ubicado a $184 \mathrm{Km}$ de la capital del país, es un municipio de 28.310 habitantes (Departamento Administrativo Nacional de Estadística, 2005) y con una población flotante de cinco mil habitantes.

Para enmarcar esta experiencia en lo tecnológico, es necesario tomar como referencia lo estipulado por la Unión Internacional de Telecomunicaciones (2004), Cumbre Mundial sobre la sociedad de la información Ginebra (2003) - Túnez (2005), concretamente en el plan de acción, estrategias C1 y C3 en donde se define el papel de los gobiernos y todas las partes interesadas en la promoción de las Tecnologías de la Información y las Comunicaciones (TIC), para el desarrollo y la importancia del acceso a la información y al conocimiento; en estas estrategias, se despliega la importancia de las TIC definiendo claramente que la población debe tener acceso a la información y al conocimiento en cualquier lugar del mundo y de manera prácticamente instantánea.

También es necesario considerar que el Estado colombiano establece en el Decreto presidencial 1151 de abril 14 de 2008 los lineamientos generales de la estrategia denominada Gobierno en Línea, en donde entidades territoriales a través de la plataforma www.gobiernoenlinea.gov.co, con el apoyo del Ministerio de Tecnologías de la Información y las Comunicaciones, suministran datos, trámites y en general noticias actualizadas como base del ejercicio transparente y eficaz para asegurarse del suministro de información permanente. En desarrollo de lo anterior el municipio de Paipa cuenta con la plataforma correspondiente www.paipa-boyaca.gov.co, la cual posee todas las ventajas de la tecnología informática en términos de acceso remoto a la información, recursos en línea, repetibilidad, entre otras. 
En este caso particular, el uso de la plataforma como herramienta tecnológica se convirtió en una ayuda eficiente para los funcionarios públicos del municipio, permitiendo plasmar información detallada y facilitando el seguimiento y control. De la misma manera permitió la elaboración y comunicación de planes, programas y proyectos e informes específicos para los entes de control y para el control ciudadano, a través de documentos amigablemente elaborados y con funciones básicas de Microsoft Office, facilitando el manejo de imágenes (fotografías y gráficos), hojas de cálculo o procesadores de texto convertidos posteriormente a pdf. Así, la administración pública del periodo 2008 -2011 aseguró la accesibilidad, repetibilidad y trazabilidad de la información, dispuesta para la comunidad al mínimo costo y con amplia cobertura, pues la información se encuentra al alcance de personas nacidas en Paipa residentes en otras ciudades de Colombia y el mundo.

Basado en lo anterior, es posible argumentar que realizar procesos de planeación para una ciudad de más de treinta mil habitantes exige un enfoque sistémico y habilidades para el manejo de herramientas tecnológicas de quienes coordinan el proceso de planeación, instando una toma de datos cuidadosamente ordenados para tomar decisiones; lo anterior implica que de manera estricta se documente, sistematice, organice y analice la información tendiente a priorizar la asignación de recursos para dar solución a la problemática en aspectos relacionados con el desarrollo económico, social y ambientalmente sostenible. Además, es fundamental considerar que requiere técnicas de localización geográfica y herramientas tecnológicas particulares para ordenar el territorio bajo criterios de prevención de riesgos, productividad y competitividad, situaciones que no siempre se dan en los 1123 municipios de Colombia.

Por otra parte, realizar un proceso de planeación participativa exige competencias para manejo de un importante volumen de información primaria. En el caso presentado, con el uso de herramientas básicas como Excel (originalmente diseñadas como hojas de cálculo financieros o contables) se articularon más de 500 indicadores trabajados en texto correspondientes a proyectos orientados a solucionar las necesidades del municipio, los cuales permiten además conocer el estado y avance de ejecución en los proyectos del plan de desarrollo de Paipa.

Cabe resaltar que tal cantidad de indicadores dificultaría la toma de decisiones por parte de la alta dirección, razón por la cual se priorizó y se llegó a establecer aquellos considerados críticos de desempeño, construyéndose el cuadro de mando integral (Ver figura 1) donde se organizan de abajo hacia arriba las dimensiones: Finanzas Públicas, Modernización Institucional y Gestión de Procesos Misionales y se muestra el camino a recorrer para mejorar las condiciones de calidad de vida de forma sostenible, responder de manera ágil, oportuna y con calidad a los requerimientos de la comunidad y finalmente, alcanzar la efectividad y adecuado funcionamiento con transparencia y aceptación de los ciudadanos establecidos en la dimensión denominada "Nuestra Comunidad." 


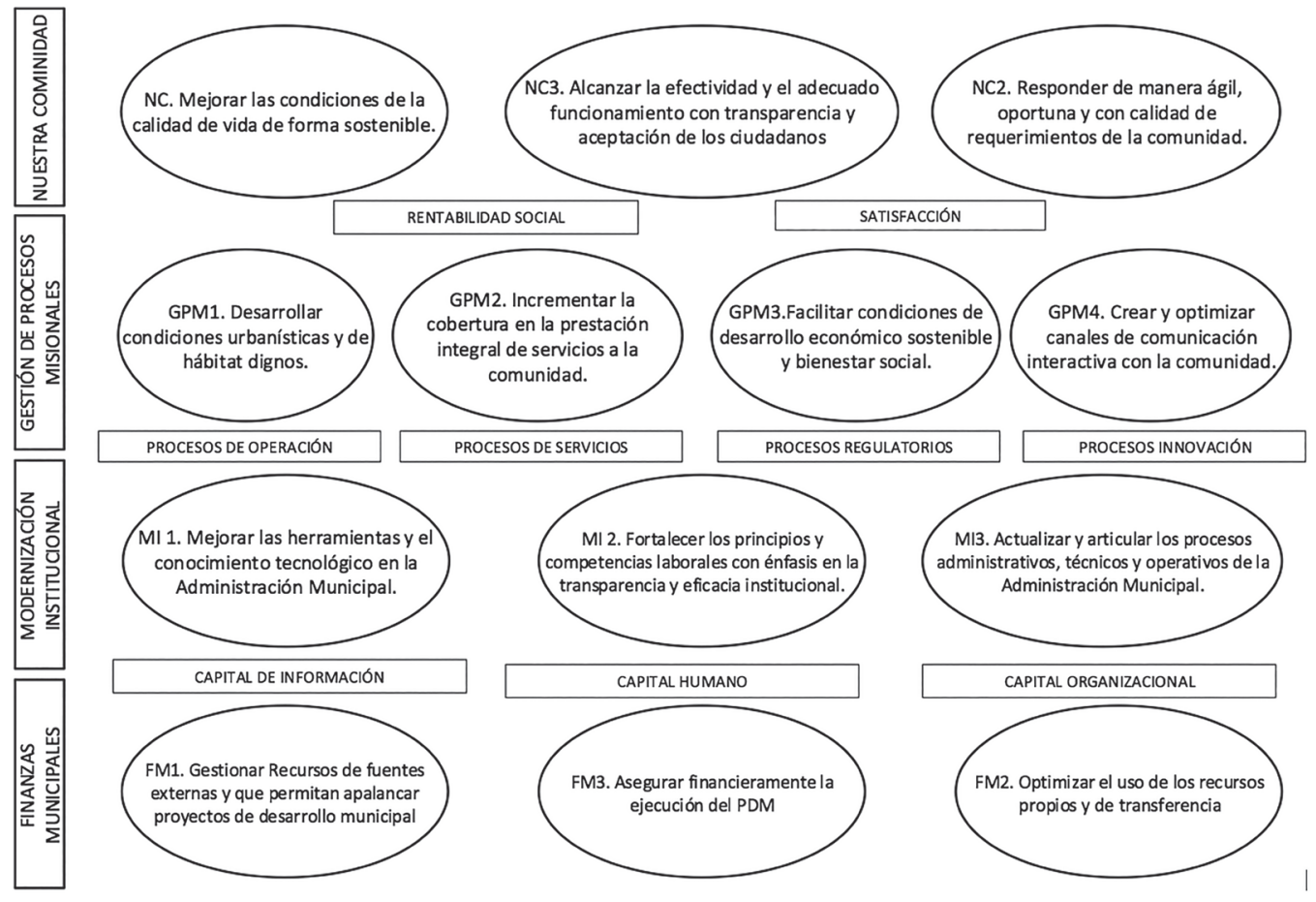

Figura 1. Dimensiones del Cuadro de mando integral PDM 2008-2011

Fuente: Planeación Municipio de Paipa (2008)

Además, es importante resaltar que la articulación del cuadro de mando con los Objetivos de Desarrollo del Milenio y la disposición de datos, facilita el proceso de comunicación ya que permite acceder desde la plataforma a información relevante de la municipalización. Este mecanismo para socializar el resultado del esfuerzo de conjunto entre el equipo ciudadano - ECODEM, funcionarios de la Alcaldía Municipal y la Agencia de Cooperación Técnica Alemana - GTZ, garantiza el monitoreo de las mejoras significativas y sostenibles en el cubrimiento de las necesidades básicas a toda la población, en especial a aquella que se encuentra en condiciones de pobreza extrema o mendicidad.

Particularmente, en el proceso de planeación se establecen metas sobre las cuales se debe ejercer posteriormente medición y control, que para el caso se alinean a los objetivos convenidos internacionalmente en la Cumbre del Milenio de las Naciones Unidas (2003) los cuales constituyen un pacto entre las naciones para eliminar la pobreza humana y reducir a la mitad la pobreza extrema y el hambre. Cada meta es medida a través de indicadores los cuales son alimentados periódicamente con el objeto de rendir cuentas a la comunidad; esta labor a pesar de ser bastante compleja se facilita con el manejo 
de programas y/o aplicaciones gratuitos que permiten construir documentos con grandes valores constitucionales y alineados a las estrategias nacionales y mundiales.

El proceso técnico de tratamiento a la información, la coherencia entre las necesidades, los programas, los proyectos, las acciones y el ejercicio participativo del plan de desarrollo municipal para el periodo 2008 - 2011, hizo acreedor al municipio al premio del quinto mejor plan de desarrollo, galardón relevante si se considera que son 1123 municipios en el país, adicionalmente generó línea base, metas, estrategias, responsables y asignación de recursos creando el punto de partida para los procesos de rendición de cuentas y aseguraron una administración pública bien direccionada.

Otra experiencia del uso de herramientas tecnológicas en lo público fue la conocida en el Centro de Formación de Talento Humano en Salud (CFTHS) del Servicio Nacional de Aprendizaje (SENA), para el manejo de información necesaria en procesos de planeación y mejora continua, asegurando la oportunidad, la comunicación, la disponibilidad y repetibilidad de la información a través de la plataforma virtual. Para enmarcar esta experiencia es necesario tener en cuenta la Ley 872 de 2003, que crea el sistema de gestión de la calidad en el sector público; desde allí, se gesta lo relativo a sistemas de calidad y se establece la Norma Técnica Colombiana de Gestión Pública- NTCGP 1000 cuyo propósito es mejorar el desempeño y capacidad de proporcionar productos y/o servicios que respondan a las necesidades y expectativas de los usuarios.

El SENA como entidad pública, cuenta con una importante herramienta tecnológica denominada Plataforma de Educación Electrónica Blackboard Professional Education Suite ${ }^{\mathrm{TM}}$ (C1997-2011) Blackboard Inc., que para el caso aquí expuesto soporta cursos virtuales. Esta plataforma es aprovechada por el equipo de Mejora del CFTHS- SENA, como soporte documental para dar cumplimiento a lo establecido en la NTCGP 1000:2009, numeral 4.2.

Basado en lo anterior, el uso de esta herramienta facilita la visualización de la cadena de valor denominada "Sistema Integrado de Mejora Continua", que contempla acciones en calidad, modelo de control interno y gestión ambiental; en dicho sistema se incluyen los procesos estratégicos o visionales o llamados "procesos de innovación", compuestos por inteligencia organizacional, planeación estratégica y mejora continua, los procesos misionales llamados "procesos de valor" que contemplan normalización de competencias laborales, diseño curricular, administración educativa, evaluación y certificación de competencias laborales, ejecución de servicios complementarios, el proceso de "ejecución y seguimiento de la formación profesional integral" y los procesos que soportan la satisfacción del cliente denominados "procesos de apoyo" que incluyen gestión del talento humano, gestión administrativa, gestión de los recursos financieros, gestión documental, gestión de la infraestructura maquinaria y equipo y gestión informática, tal y como se visualiza en la Figura 2. 


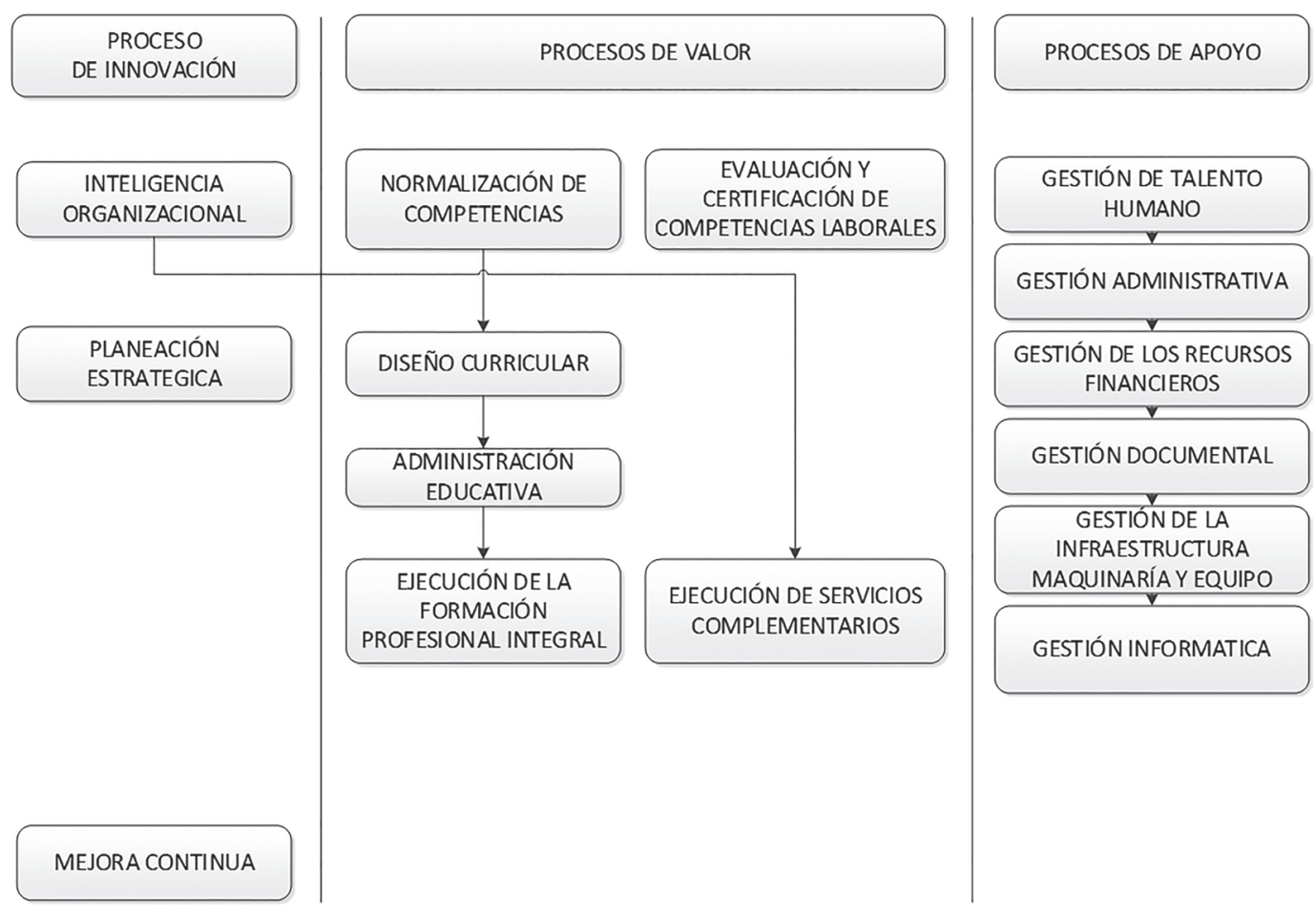

Figura 2. Cadena de Valor Sistema Integrado de Mejora Continua Fuente: CFTHS (2011).

Desde allí y acorde a los requerimientos, es posible tener acceso a la información específica; por ejemplo en el proceso denominado Mejora Continua se encuentran documentos de control como son matriz de indicadores, hoja de vida de dichos indicadores, desempeño de indicadores de gestión y procedimientos documentados exigidos por NTCGP 1000 (Control de registros, control de documentos, acciones preventivas, acciones correctivas, control de producto no conforme y auditoría interna).

La información disponible en la plataforma permite comprender el funcionamiento del CFTHS, facilita la rápida articulación al sistema, por ello se disminuyen los inconvenientes frente a los efectos de convocatorias públicas y la selección variada de profesionales, pues el sistema asegura la apropiación de la filosofía de planear, hacer, verificar y actuar, y la aplicación rigurosa en los procedimientos hace que se reduzca la variabilidad en el servicio que presta el CFTHS de todos los procesos de su cadena de valor. 
Asimismo, el funcionario puede hacer uso del correo electrónico dominio denominado "sena" y "misena" es decir, ejemplo@sena.edu.co y ejemplo@misena.edu.co para optimizar el proceso de comunicación en la cultura "cero papel”, el correo electrónico se considera comunicación oficial desde el dominio sena.edu.co, convirtiéndose esta herramienta tecnológica en evidencia de las acciones de mejora.

Es de indicar que la información es controlada por el líder del proceso de gestión documental quien hace parte del equipo de mejora continua y del comité de calidad, este líder posee formación, conocimiento, experiencia y habilidades en lo documental y en el manejo de programas y aplicaciones que ayudan a generar cambios culturales, no solo para el aprendiz sino también para el cuidado del medio ambiente y el control interno.

Finalmente, la herramienta tecnológica sirve para suministrar información constante, sin barreras de espacio y tiempo para la correcta ejecución de los procesos; permite el control en forma permanente de documentos, registros, acciones correctivas, acciones preventivas, procedimientos de retención o liberación de producto no conforme, seguimiento desde la Alta Dirección, control y aseguramiento del servicio que redunda en el reconocimiento y la recertificación del CFTHS por parte del ente certificador.

\section{LA ARTICULACIÓN CON LA INGENIERÍA INDUSTRIAL}

Partiendo del concepto del Instituto de Ingenieros Industriales (2012), que dice que "es al Ingeniero Industrial a quien le concierne el diseño, mejora, e instalación de sistemas integrados por personas, material, información, equipo y energía” y considerando lo definido por la Asociación Colombiana de Facultades de Ingeniería - ACOFI (2010), quien expone su propósito hacia la Ingeniería Industrial como "formar profesionales que apliquen sus conocimientos y habilidades para el diseño, planeación, gestión, optimización y control de sistemas de producción de bienes y servicios en organizaciones o en sistemas complejos, los cuales involucran personas así como recursos financieros, técnicos, materiales y de información en búsqueda de lograr incrementar los indicadores de desempeño", es posible pensar entonces que quienes ejercen la Ingeniería Industrial deben estar en capacidad de abordar desafíos de administración de entidades, sin importar si son públicas o privadas. Igualmente para los Ingenieros Industriales ejercer la profesión en temas relacionados con la gestión administrativa y desempeñarse en entidades que administran recursos del Estado, exige mayor creatividad e innovación en lo relacionado al uso de las herramientas tecnológicas disponibles en el mercado para la recopilación, tratamiento y generación de reportes que suministren datos requeridos por la alta dirección y que faciliten la toma de decisiones.

Desde este punto de vista y considerando la contratación pública, es imprescindible contemplar múltiples restricciones en variables de tiempo, costo, normatividad legal, procesos y procedimientos dentro 
del proceso de compra de software especializados, lo cual implica mayor cantidad de habilidades para procesos de diseño, planeación, optimización, control y comunicación.

El reto está en entender bajo un enfoque sistémico la situación de una entidad pública y optimizar procesos, aplicando nuevas metodologías para priorizar proyectos apoyados en los recursos técnicos y tecnológicos; se requiere además demostrar competencia para coordinar la interdisciplinariedad presente en el talento humano de las organizaciones, por ello los esfuerzos deben centrarse en buscar desencadenar la participación disciplinar (ciencias humanas, administrativas, técnicas, entre otras) bajo un único lenguaje de efectividad y transparencia en el actuar público.

Los Ingenieros Industriales son los profesionales llamados a buscar y asegurar que la disponibilidad y repetibilidad de la información permita planear nuevos desarrollos, que solo desde la planeación en lo público se puede lograr, como es el caso de acciones en ciudad, siendo fundamental el trabajo interdisciplinar (por los requerimientos simultáneos en formulación y ejecución de proyectos, en términos ambientales, legales, financieros, culturales, de servicios públicos y de desarrollo urbano), la cooperación y la participación comunitaria.

Entender los procesos y sistemas requiere del Ingeniero Industrial una nueva capacidad para hacer sencillos y prácticos los procesos, utilizando el mínimo de recursos con el máximo de cobertura, maximizando el uso de herramientas tecnológicas en lo relacionado con tratamiento de información.

Se evidencia que el adecuado uso de diferentes herramientas tecnológicas facilita el proceso de planeación como base del mejoramiento continuo, ya que permite el seguimiento y monitoreo de recursos asignados a las estrategias planteadas inicialmente. El uso de estas herramientas en entidades públicas como CFTHS, son fundamentales en el proceso de comunicación oportuna y eficiente, eliminan barreras entre el Estado y la comunidad y se convierten en el soporte documental del Sistema de Gestión de la Calidad, para procesos de Auditoria.

Las herramientas tecnológicas en las organizaciones públicas son ahora el punto de encuentro para los equipos interdisciplinarios y es el Ingeniero Industrial quien debe actuar como coordinador y orientar esfuerzos del equipo hacia el mejor aprovechamiento de los recursos, potencializar y acelerar el logro de resultados con procesos y sistemas holísticos. 


\section{CONCLUSIONES}

Desde la perspectiva de la Ingeniería Industrial, la gestión de lo público coincide perfectamente con su propósito de maximizar resultados con diversas restricciones, exigiendo el manejo de situaciones complejas en donde los recursos que en apariencia son suficientes, en el fondo y dadas las restricciones legales para su ejecución terminan por ser insuficientes ante la cantidad de necesidades de la población. Se evidencia que el adecuado uso de diferentes herramientas tecnológicas facilita el proceso de planeación como base del mejoramiento continuo, ya que permite el seguimiento y monitoreo de recursos asignados a las estrategias planteadas inicialmente, debido a que es posible controlar desde la línea base o estado actual los avances planteados en las metas mediante indicadores de eficacia y eficiencia, entre otros.

El uso de herramientas tecnológicas por entidades públicas como CFTHS es fundamental en el proceso de comunicación oportuna y eficiente, elimina barreras entre el Estado y la comunidad en general; facilitando el acceso a la información, convirtiéndose en soporte documental para el Sistema de Gestión de la Calidad, herramienta prioritaria para los procesos de Auditoria de la entidad.

La interacción con profesionales de Salud en el CFTHS del SENA, especialmente en lo relacionado con uso e innovación de herramientas tecnológicas en el soporte documental del Sistema Integrado de Mejora Continua, representa innovación y aprendizaje.

El ejercicio de la Ingeniería Industrial encuentra en el sector público un campo fértil que contribuye al desarrollo socioeconómico del país, no solo por la necesidad de diseñar e implementar procesos y procedimientos eficientes y eficaces, sino por soportar procesos de optimización de recursos, manejo de indicadores y toma de decisiones. 


\section{REFERENCIAS BIBLIOGRÁFICAS}

Alcaldía Municipal de Paipa.(2011). Plan de Desarrollo 2008-2011“En Paipa Primero la Gente”. Paipa, Colombia.

Asociación Colombiana de Facultades de Ingeniería. (2009). Prospectiva de la ingeniería industrial en Colombia al 2020 - "Retos y Desafíos". Bogotá - Colombia.

Alzate Gómez José Ángel (2009), Capital social, descentralización y modernización del estado. Recuperado de http://www.eumed.net/libros-gratis/

Congreso de Colombia. (2003) Ley 872 Por la cual se crea el sistema de gestión de la calidad en la rama ejecutiva del poder público y en otras entidades prestadoras de servicios. Bogotá, Colombia.

Consejo Profesional de Ingeniería COPNIA (2009). concepto NAL-CE-2009-00611. Bogotá, Colombia. Recuperado de https://copnia.gov.co

Departamento Administrativo de la Función Pública (2014). Gestión pública local. Bogotá, Colombia. Recuperado de https://www.dafp.gov.co

Departamento Nacional de Estadística. (2005). Proyecciones de población, Recuperado de https:// www.dane.gov.co/index.php/es/poblacion-y-registros-vitales.

Deming, W.E. (1982). Calidad, productividad y competitividad: la salida de la crisis. España. Diaz Santos

Departamento Nacional de Planeación (2005). Consejo Nacional de Política Económica y Social. Bogotá, Colombia.

Departamento Nacional de Planeación (2014). Atención al ciudadano. Bogotá, Colombia. Recuperado de https://www.dnp.gov.co

Escorsa, C \& Valls Jaume. (2005). Tecnología e innovación en la Empresa. Recuperado de http:// www. gcd.udc.es/

Escuela Superior de Administración Pública (2014), Especialización Gestión y Planificación del Desarrollo Urbano y Regional. Bogotá, Colombia. Recuperado de http://www.esap.edu.co/

Instituto Colombiano de Normas Técnicas (2009). Norma Técnica de Calidad en la Gestión Pública. NTCGP 1000:2009. Bogotá, Colombia.

Instituto de Ingenieros Industriales (2012). Recuperado de http://www.iienet2.org 
Jaramillo, L. J (1985). Serie Aprender a Investigar. Instituto Colombiano para el Fomento de la Educación Superior. Bogotá, Colombia.

Ministerio del Interior y de Justicia (2008). Decreto Ley 1151. Recuperado de http//www.programa. gobiernoenlinea.gov.co.

Núñez de Schilling, Elizabeth. Gestión tecnológica en la empresa: definición de sus objetivos fundamentalesRevista de Ciencias Sociales (Ve) [en linea] 2011, XVII (Enero-Marzo): [Fecha de consulta: 21 de julio de 2014] Disponible en:<http://www.redalyc.org/articulo.oa?id=28022755013> ISSN $1315-9518$

Programa de Naciones Unidas para el Desarrollo (2000). Los Objetivos de Desarrollo del Milenio:La Agenda Global para superar la Pobreza. Recuperado de http://www.pnud.org

Rincon, A \& Ocampo, E.(2010) Prospectiva de la ingeniería industrial en Colombia al 2020 "retos y desafíos". Asociación Colombiana de Facultades de Ingeniería. Bogotá. Colombia

Unión Internacional de Telecomunicaciones. (2004) Cumbre Mundial sobre la sociedad de la información Ginebra 2003 - Túnez 2005. Recuperado de (http://www.itu.int/wsis/docs/geneva/official/ poa-es.html)",

Rincon, A \& Ocampo, E.(2010) Prospectiva de la ingeniería industrial en Colombia al 2020 "retos y desafíos". Asociación Colombiana de Facultades de Ingeniería. Bogotá. Colombia

Sequera,L., \& Grelys M (2010). Competencias básicas en el uso de herramientas tecnológicas: un reto para los profesores asesores del programa nacional de formación. En G. Sequera Ladino. Congreso Internacional de Educación Superior. La Habana, Cuba.

Servicio Nacional de Aprendizaje, Centro de Formación Talento Humano en Salud.(2011). Comunidad de aprendizaje. Recuperado de https://senastagesp4.blackboard.com

Troconiz, D. (2007). Ingeniería Industrial. Argentina: El Cid.

Entidades vinculadas.

Caso Paipa - Alcaldía Municipal - Equipo ciudadano - ECODEM - GTZ - Boyacá Digital

Caso SENA - Centro de Formación de Talento Humano en Salud - CFTHS

Personas Vinculadas.

A continuación se presenta los nombres de las personas directamente involucradas en procesos de los casos aquí expuestos 


\section{Caso Paipa}

Alcalde Municipal - Jorge Alberto Herrera

Asesor GTZ para el Municipio - Felipe Melgarejo

Responsable Boyacá Digital - Municipio de Paipa. - Aida Consuelo Castro

\section{Caso SENA}

Comité de Calidad CFTHS - SENA

Subdirector de Centro - Gerardo Arturo Medina R.

Representante de la Dirección: María Ligia Cruz

Líderes de Procesos: Susana García de Pabón, Jairo Rizo, Claudia Gómez, Juan Carlos Alarcón, Ruth Mireya Suarez. Líderes de Proceso, Miembros del Equipo de Mejora Continua y Autoras del Sistema - Claudia H. Becerra y Gilma Janneth Guativa 